\title{
A teoria Nightingaleana e seu legado na prevenção das infecções cirúrgicas no Brasil: testando tecnologia
}

The Nightingalean theory and its legacy in the prevention of surgical infections in Brazil: testing technology

La teoría de Nightingalean y su legado en la prevención de infecciones quirúrgicas en Brasil: tecnología de pruebas

Jackeline Franco Couto

ORCID: https://orcid.org/0000-0002-7720-0102 Universidade Federal do Estado do Rio de Janeiro, Brasil

E-mail: jack.enfa@hotmail.com

Wiliam César Alves Machado

ORCID: https://orcid.org/0000-0002-4325-7143 Universidade Federal do Estado do Rio de Janeiro, Brasil E-mail: wily.machado@gmail.com

Eva Maria Costa

ORCID: https://orcid.org/0000-0002-0318-5587 Universidade Federal do Rio de Janeiro, Brasil E-mail: evamariacosta@ig.com.br

Maria Antonieta Rúbio Tyrrel

ORCID: https://orcid.org/0000-0002-1885-6865

Universidade Federal do Rio de Janeiro, Brasil E-mail: tyrrel12004@hotmail.com

Kátia Maria dos Santos Calegaro ORCID: https://orcid.org/0000-0001-9641-7246 Conselho Regional de Enfermagem do Rio de Janeiro, Brasil E-mail: katiacalegaro@gmail.com

Ludmila Santos Oliveira

ORCID: https://orcid.org/0000-0002-9701-5318 Conselho Regional de Enfermagem do Rio de Janeiro, Brasil E-mail: nursemila@yahoo.com.br

Nébia Maria Almeida de Figueiredo

ORCID: https://orcid.org/0000-0003-0880-687X Universidade Federal do Estado do Rio de Janeiro, Brasil E-mail: nebia43@gmail.com

\begin{abstract}
Resumo
O objetivo deste artigo é refletir sobre as contribuições do legado deixado por Florence Nightingale, através de sua teoria ambientalista, discutindo enfoques conceituais, teóricos e práticos para as políticas de prevenção e controle das infecções cirúrgicas no Brasil e no empoderamento do Enfermeiro, através da testagem de uma tecnologia de desinfecção de ambiente. Método: trata-se de uma reflexão teórica, baseada em uma pesquisa bibliográfica para fundamentação teórica, com abordagem qualitativa. Resultado: a teoria ambientalista desenvolvida por Florence Nightingale tem sido amplamente utilizada pelos estabelecimentos assistenciais de saúde, desde a idade antiga até os dias atuais. O Enfermeiro tem a total capacidade de realizar testagem com tecnologias, no entanto necessita se capacitar e imergir na área da temática da pesquisa, além de se comprometer em desenvolver com rigor todas as etapas envolvidas no estudo, mas também precisa contar com a disponibilização de recursos financeiros, que necessitam ser injetados nas universidades públicas, instituições e institutos de pesquisa, direcionados às pesquisas científicas no Brasil. Conclusão: Florence Nightingale permanece presente com seus preciosos ensinamentos para Enfermeiros e demais profissionais da saúde e de outras áreas, suas contribuições vão de encontro com a prevenção e controle das infecções relacionadas a assistência à saúde, em especial, as infecções cirúrgicas. Nesse ínterim, o Enfermeiro é peça fundamental do cuidado capaz de testar uma tecnologia de desinfecção no ambiente cirúrgico à base de Radiação ultravioleta-c.
\end{abstract}

Palavras-chave: Teoria de enfermagem; Infecção da ferida cirúrgica; Controle de infecções; Enfermeiros; Tecnologia. 


\begin{abstract}
The objective of this article is to reflect on the contributions of the legacy left by Florence Nightingale, through his environmental theory, discussing conceptual, theoretical, and practical approaches to the policies for the prevention and control of surgical infections in Brazil and the empowerment of Nurses, through testing environment disinfection technology. Method: it is a theoretical reflection, based on bibliographic research for theoretical foundation, with a qualitative approach. Result: the environmental theory developed by Florence Nightingale has been widely used by health care establishments from ancient times to the present day. The nurse has the full capacity to carry out testing with technologies. However, they need to be trained and immerse themselves in the area of the research, in addition to committing themselves to develop all the stages involved in the study rigorously. Still, they also need to have financial resources, which need to be injected into public universities, institutions, and research institutes, aimed at scientific research in Brazil. Conclusion: Florence Nightingale remains present with her precious teachings for Nurses and other health professionals and other areas. Her contributions are in line with the prevention and control of healthcare infections, especially surgical infections. In the meantime, the nurse is a fundamental part of the care capable of testing a disinfection technology in the surgical environment based on ultraviolet C-radiation.
\end{abstract}

Keywords: Nursing theory; Surgical wound infection; Infection control; Nurses male; Technology.

\title{
Resumen
}

El objetivo de este artículo es reflexionar sobre las contribuciones del legado dejado por Florence Nightingale, a través de su teoría ambiental, discutiendo enfoques conceptuales, teóricos y prácticos de las políticas de prevención y control de infecciones quirúrgicas en Brasil y el empoderamiento de las enfermeras, a través de Prueba de tecnología de desinfección del entorno. Método: es una reflexión teórica, basada en una investigación bibliográfica para fundamento teórico, con un enfoque cualitativo. Resultado: la teoría ambiental desarrollada por Florence Nightingale ha sido ampliamente utilizada por los establecimientos de salud, desde la antigüedad hasta nuestros días. La enfermera tiene plena capacidad para realizar pruebas con tecnologías, sin embargo necesita capacitarse y sumergirse en el área del tema de investigación, además de comprometerse a desarrollar rigurosamente todas las etapas involucradas en el estudio, pero también necesita contar con la disponibilidad de recursos. financieros, que deben inyectarse en universidades públicas, instituciones e institutos de investigación, destinados a la investigación científica en Brasil. Conclusión: Florence Nightingale permanece presente con sus preciadas enseñanzas para Enfermeras y otros profesionales de la salud y otras áreas, sus aportes están en línea con la prevención y control de infecciones relacionadas con la salud, especialmente las quirúrgicas. Mientras tanto, la enfermera es una parte fundamental del cuidado capaz de probar una tecnología de desinfección en el entorno quirúrgico basada en radiación ultravioleta C.

Palabras clave: Teoría de enfermería; Infección de heridas quirúrgicas; Control de infección; Enfermeras; Tecnología.

\section{Introdução}

Desde os ensinamentos propostos pela Mentora da Teoria Ambientalista, Florence Nightingale, a prevenção de Infecções Relacionadas a Assistência à Saúde (IRAS) vem sendo uma crescente preocupação mundial dos profissionais atuantes nos estabelecimentos assistenciais de saúde, por se tratar de um indicador que interfere diretamente na qualidade da assistência ao paciente.

Florence Nightingale tem sido reverenciada há décadas por suas contribuições para o conhecimento, educação e práticas da Enfermagem, bem como para as reformas da atenção à saúde. Ela foi pioneira na aplicação da Epidemiologia e Estatística no campo do cuidado à saúde. No caso da Estatística, considerava-a essencial para entender os problemas sociais e procurou introduzir o estudo desse ramo do conhecimento para justificar suas conclusões no campo da saúde, por exemplo, utilizando a ilustração gráfica para apresentar os resultados de suas pesquisas" (Carraro, 2004).

A teoria ambientalista desenvolvida por Florence Nightingale, no final do primeiro terço do século XIX tinha, como foco principal, o meio ambiente. A prática de intervenção na saúde se constituía de um conjunto de ações que auxiliassem a natureza a promover a cura do doente. Florence afirmava que a Enfermagem era uma prática que buscava colocar o paciente na melhor condição para a ação da natureza (Castro, 1989). Segundo Florence, as áreas principais de controle ambiental envolviam a ventilação, o calor, as emanações (odores), o barulho e a iluminação (McEwen \& Wills, 2009).

Historicamente, no Brasil, a demanda pelo controle e prevenção das IRAS, inicialmente denominada infecção hospitalar (IH), se deu em meados dos anos 70 do século XX, por recomendação do Ministério da Previdência e Assistência 
Social (MPAS), a partir de profissionais que já estudavam e lidavam com esse tipo de ocorrência no país, e que havia criado as primeiras Comissões de Controle e Prevenção de IH (CCIH) nos hospitais em que trabalhavam (Ministério da Saúde, 1998).

As Infecções do Sítio Cirúrgico (ISC) são as complicações mais comuns decorrentes do ato cirúrgico, que ocorrem no pós-operatório em cerca de 3 a 20\% dos procedimentos realizados, tendo um impacto significativo na morbidade e mortalidade do paciente. As ISC são consideradas eventos adversos frequentes, decorrente da assistência à saúde dos pacientes que pode resultar em dano físico, social e/ou psicológico do indivíduo, sendo uma ameaça à segurança do paciente (Agência Nacional de Vigilância Sanitária, 2017).

No Brasil, a Infecção de Sítio Cirúrgico (ISC) ocupa a $3^{\mathrm{a}}$ posição, com cerca de 14\% a 16\% das infecções encontradas em pacientes hospitalizados. Nos Estados Unidos da América, estima-se a ocorrência de 150.000 a 300.000 Infecções do Sítio Cirúrgico, que são responsáveis por 8,205 óbitos anuais (Agência Nacional de Vigilância Sanitária, 2017).

$\mathrm{Na}$ área da saúde, assim como nas áreas da educação, das ciências exatas, dentre outras, as demandas para a invenção e aperfeiçoamento de artefatos tecnológicos, seguido de um avanço nas tecnologias tem sido cada dia mais evidenciadas.

Como ferramenta de estratégias de prevenção das infecções cirúrgicas, o estudo propõe uma reflexão realizada com base nos ensinamentos deixados por Florence Nightingale e dos resultados após testagem de tecnologia de desinfecção em ambiente cirúrgico, de um hospital universitário do Rio de Janeiro. Nesse contexto, surge a seguinte questão norteadora de pesquisa: "Pode a teoria ambientalista contribuir e ser aplicada na Prevenção e Controle das Infecções Cirúrgicas no Brasil"?

\section{Metodologia}

Trata-se de uma reflexão teórica, baseada em uma pesquisa bibliográfica para fundamentação teórica, com abordagem qualitativa, fundamentada na Teoria Ambientalista de Florence Nightingale e na formulação discursiva acerca da testagem de uma tecnologia de desinfecção de ambiente, em prol da prevenção e controle das infecções cirúrgicas no Brasil.

O estudo reflexivo está pautado na seguinte questão norteadora: Pode a teoria ambientalista contribuir e ser aplicada na Prevenção e Controle das Infecções Cirúrgicas no Brasil?

Assim, foram utilizadas como fonte de dados a literatura científica sobre a Teoria Ambientalista de Nightingale, com vistas a amparar a discussão e a análise crítica, abrangendo livros, resoluções e artigos científicos, coletados em bases de dados online que tivessem interface com o tema.

\section{Resultados e Discussão}

3.1 Trilhando caminhos na Prevenção das Infecções do Sítio Cirúrgico, em Busca de um Ambiente Seguro nos Estabelecimentos Assistenciais de Saúde (EAS)

Com base numa retrospectiva histórica, constatamos ao longo dos séculos, que a teórica Florence Nightingale direcionou a sua preocupação em busca da promoção de um ambiente saudável e sua representatividade serve de parâmetro desde a idade antiga até os dias atuais.

Os conceitos recentes de segurança do paciente da Organização Mundial de Saúde (OMS) remetem aos Princípios Nightingaleanos, uma vez que a ocorrência de danos e prejuízos associados aos cuidados em saúde é decorrente de falhas nos processos ou estruturas assistenciais que, muitas vezes, fogem da governabilidade de enfermeiros no Brasil, enquanto Nightingale, opinava e se envolvia nas modificações das plantas hospitalares em prol da melhoria da oferta de cuidados.

A representatividade teórica da obra de Florence envolve diretrizes para enfermeiros e profissionais de outras áreas do conhecimento. Naquela época, Nightingale escreveu a importância a respeito das vantagens da recuperação e dos efeitos 
imediatos da cirurgia em uma unidade separada do hospital, observando seu cuidado de evitar infecções cruzadas vindas de outros pacientes enfermos e um cuidado melhor assistido (Matarazzo, 2013).

Nessa perspectiva, um estudo realizado em 2017 no Brasil, evidenciou que Florence descreveu dezoito itens que elencava como fundamentais para a construção de um hospital, como os números de pavimentos e de alas ou unidades por pavilhão, o dimensionamento das alas ou unidades, o espaçamento entre leitos, a relação entre leitos e janelas, materiais de construção e partido arquitetônico, as salas de enfermagem e localização, casa de banho, os lavatórios, vasos sanitários e pia para lavagem de materiais, ventilação natural, mobiliário da unidade do paciente, leito, tratamento da água, sistemas de esgoto, cozinhas e alimentos, lavanderia e centro cirúrgico (Draganov \& Sanna, 2017).

No país, no decorrer de 30 anos, diversas foram as ações governamentais desenvolvidas em prol da prevenção e controle de Infecções Relacionadas à Assistência à Saúde (IRAS) nos Estabelecimentos Assistenciais de Saúde (EAS), em busca da segurança dos pacientes nos serviços de saúde.

As recomendações descritas na Resolução de Diretoria Colegiada (RDC) $\mathrm{n}^{\circ}$ 50, de 21 de fevereiro de 2002 (Ministério da Saúde, 2002), que dispõe sobre o Regulamento Técnico para planejamento, programação, elaboração e avaliação de projetos físicos de estabelecimentos assistenciais de saúde, têm sido associadas à segurança dos pacientes e à saúde do trabalhador, desde a sua criação no Brasil.

Vale destacar, que essa resolução supracitada apresenta relação direta com a Teoria Ambientalista da precursora da Enfermagem moderna, Florence Nightingale, que já descrevia, à sua época, como deveria ser elaborada uma estrutura hospitalar, o cuidado com o ambiente e sua preocupação com a Prevenção e Controle de Infecções, apesar de não contar com os avanços tecnológicos. Nesse sentido, são descritas na Resolução de Diretoria Colegiada no 50/2002 (Ministério da Saúde, 2002), as recomendações básicas sobre a estrutura física no centro cirúrgico, tais como: Sistema de ventilação, circulações externas e internas, circulações verticais, monta-cargas, tubo de queda, condições ambientais de controle de infecção, colocação de lavatórios/ pias/ lavabos cirúrgicos, acabamentos de paredes, pisos, tetos e bancada, rodapés e forros.

É inegável, que Florence Nightingale traçou caminhos e modelos assistenciais a serem seguidos na atualidade, mas acreditamos que esses modelos sofrem constantemente influências do contexto político social vigente, e as adaptações se fazem de forma a atender essas influências. É diante dessa realidade que a enfermagem traça seu caminho e estabelece seu pensar e seu fazer.

O Enfermeiro tem um papel fundamental na prevenção e controle de infecções cirúrgicas, que envolve conhecimentos científicos teóricos e habilidades práticas, para a obtenção de uma assistência livre de danos aos pacientes.

Machado e Figueiredo, 2012 consideram a perspectiva ambientalista teorizada por Florence Nightingale quando descrevem os "Cenários do Cuidar" como sendo um local onde:

[...] as cenas de cuidar são montadas e constituídas de materiais, procedimentos e profissionais específicos para cada situação, cada necessidade, cada história, cada cultura que envolve o sujeito que deve ser cuidado. A enfermagem necessita de diversos recursos para desenvolver suas ações e suas falas e, como no teatro, precisa moldar a iluminação, utilizar objetos "cênicos" plástico visuais, quiçá "figurinos", bem como tratar o espaço para cada cliente também no que diz respeito à ventilação, ao som e à aparência de limpeza e higiene do ambiente intelectual, científico e empírico daqueles que cuidam de seres humanos.

\subsection{O Empoderamento do Enfermeiro na Testagem de Tecnologia de Desinfecção à Base de Radiação Ultravioleta-C}

Para os profissionais da enfermagem, acostumados a pensar no ambiente como um espaço mínimo, onde o doente está (leito, móveis necessários e suas coisas particulares), reinterpretar Nightingale sem perdê-la de vista, está no passado e se movimenta sempre que usamos o dispositivo de chamar Nightingale. 
O Enfermeiro é peça fundamental do cuidado; com isso, é indiscutível e relevante a realização de pesquisas científicas abordando aspectos teórico-práticos. Reforçando essa ideia, foi criado um protótipo de tecnologia para desinfecção de ambiente cirúrgico à base de radiação ultravioleta-c como produto e fruto de uma Tese de Doutorado, do Curso de Pós Graduação em Enfermagem e Biociências, da Universidade Federal do Estado do Rio de Janeiro- UNIRIO (Couto, 2020), essa tecnologia foi testada numa sala cirúrgica de um hospital público universitário do Rio de Janeiro.

Os dados relacionados à testagem da tecnologia foram obtidos através do estudo observacional, do tipo seccional, no período de abril a junho de 2019, esta testagem envolveu dois cenários, o ambiente cirúrgico hospitalar e o setor de bacteriologia de um Laboratório do Rio de Janeiro (Couto, 2020).

Na pesquisa foram utilizadas três superfícies no ambiente cirúrgico e envolveram seis momentos e dois testes foram feitos com o protótipo no ambiente cirúrgico, cada dia de testagem resultou em 15 swabs coletados, que durante a pesquisa de bancada se multiplicavam em 45 placas de Petri, com meios de isolamento de culturas voltados às bactérias (Couto, 2020).

Os resultados apontaram que o Enfermeiro tem a total capacidade de realizar testagem com tecnologias, no entanto necessita se capacitar e imergir na área da temática da pesquisa, além de se comprometer em desenvolver com rigor todas as etapas envolvidas no estudo, mas também precisa contar com a disponibilização de recursos financeiros, que necessitam ser injetados nas universidades públicas, instituições e institutos de pesquisa, direcionados às pesquisas científicas no Brasil.

Vale relembrar que o ambiente e seus elementos persistem no pensamento e na prática dos Enfermeiros, que jamais poderão ser "robôs" substituídos, porque eles são e serão os vigilantes do cuidado, do ambiente e dos clientes, legado deixado por Florence Nightingale.

\section{Conclusão}

Desse modo, o simbolismo de Florence Nightingale permanece presente nos hospitais desde meados do século XVIII até o século XXI; são exatamente 200 anos de ensinamentos para a Enfermagem brasileira e mundial e não poderíamos deixar de descrever essa proximidade entre os ensinamentos deixados por Florence e as Políticas de Prevenção e Controle de Infecções Relacionadas à Assistência (IRAS), com foco nas infecções cirúrgicas no país.

Contudo, apesar dos avanços tecnológicos, como exemplo, a prática de cirurgias robóticas, percebe-se que há uma luta incansável em busca da prevenção e controle de infecções nos Estabelecimentos Assistenciais de Saúde (EAS), em especial as infecções cirúrgicas, visto que os desafios perpassam a conscientização dos profissionais de saúde, na realização de medidas básicas de prevenção e controle de infecções relacionadas a assistência à saúde, como a realização da higienização das mãos. Contudo, trata-se de um ciclo de variáveis contribuintes, de problemas não solucionados, como infraestrutura inadequada nos hospitais, falta de aquisição de recursos materiais e humanos adequados, falta de envolvimento de gestores, ações governamentais efetivas, falta de apoio financeiro, fatores estes que corroboram para a não implementação das políticas de prevenção e controle de infecções cirúrgicas no Brasil.

A Enfermagem é uma profissão que vem construindo bravamente sua história ao longo do tempo, questionando, enquanto ciência, as razões que levam a uma atuação ainda subalterna e invisível no cotidiano da prática profissional. A Enfermagem precisa estar continuamente lutando por espaços de reconhecimento de sua importância pela equipe multiprofissional e pela sociedade como um todo, por uma identidade profissional calcada na expertise e na cientificidade de suas ações e para a redução do fosso que ainda existe entre a teoria e a prática (Nelson \& Gordon, 2006).

As enfermeiras devem continuar a serem, sim, parte da solução dos desafios de saúde que a sociedade enfrenta, indo ao encontro de suas vozes individuais, tornando-as coletivas e vivendo literalmente o conceito do Nursing Now para além da campanha. Deste modo, a expertise poderá ser utilizada em prol de um benefício maior para as lutas da profissão, e que o reconhecimento da sociedade não se perca. A história continuará a ser a nossa referência, e os preceitos de Florence 
Nightingale e outros modelos fortalecerão o nosso compromisso como o conhecimento, com a nossa identidade profissional para melhor enfrentar as mudanças globais, e suas implicações para a saúde pública (Padilha, 2020).

Diante disso, sugere-se a realização de pesquisas que possam fortalecer as inúmeras contribuições do legado deixado por Florence Nightingale para a Enfermagem e demais áreas da saúde e no empoderamento do papel do Enfermeiro, através da sua inserção na prática de testagens de tecnologias de desinfecção de ambiente, que atualmente podem ser utilizadas, além de ser um tema relevante no combate ao vírus SARS-coV-2.

Para finalizar, disponibilizamos essa reflexão em que Machado e Figueiredo (2012) descrevem como exigência para o cuidado em saúde escutar e ver:

[...] o ser na sua totalidade, inclusive em relação às experiências vividas, como as de estar doente e ser cuidado pela enfermagem. Cuidar em uma perspectiva que focalize o cliente como pessoa, visando à compreensão deste em sua complexidade (história de vida, cultura, estrutura profissional/ocupacional, afetividade e espiritualidade).

\section{Referências}

Agência Nacional de Vigilância Sanitária. (2017). Medidas de Prevenção de Infecção Relacionada à Assistência à Saúde. http://portal.anvisa.gov.br/documents/33852/3507912/Caderno+4+-+Medidas+de+Preven\%C3\%A7\%C3\%A 3o+de+Infec\%C3 $\% \mathrm{~A} 7 \% \mathrm{C} 3 \% \mathrm{~A} 3 \mathrm{o}+$ Relacionada+\%C3\%A0+Assist $\% \mathrm{C} 3 \%$ AAncia+\%C3\%A0+Sa\%C3\%BAde/a3f23dfb-2c54-4e64-881c-fccf9220c373

Carraro, T. E. (2004). Os postulados de Nightingale e Semmelweis: poder/vital e prevenção/contágio como estratégias para a evitabilidade das infecções. Revista Latino-Americana de Enfermagem, 12(4), 650-657. https://doi.org/10.1590/S0104-11692004000400011

Castro, L. B. (1989). Prefácio à edição brasileira. Em F. Nightingale, Notas sobre enfermagem: o que é e o que não é (A. C. Carvalho, Trad., pp. 3-8). Cortez.

Couto, J. F. (2020). Testagem de uma tecnologia de desinfecção à base de radiação ultravioleta-c no ambiente cirúrgico: Uma metodologia para enfermeiros na perspectiva de Florence Nightingale. (p. 186). Rio de Janeiro: Universidade Federal do Estado do Rio de Janeiro.

Couto, J. F., Tyrrel, M., Araújo, S. C., Machado, W. C., \& Figueiredo, N. M. (2020). Trazendo Nightingale para o século XXI: Retrospectiva do cuidado de Enfermagem na perspectiva da Teoria Ambientalista. Research, Society and Development, 9(5). http://dx.doi.org/10.33448/rsd-v9i5.3122

Draganov, P. B., \& Sanna, M. C. (2017). Desenhos arquitetônicos de hospitais descritos no livro "Notes on Hospitals" de Florence Nightingale. História da Enfermagem Revista Eletrônica, 94-105.

Machado, W. C., \& Figueiredo, N. M. (2012). Tratado de Cuidados de Enfermagem - Médico Cirúrgico. Roca.

Matarazzo, A. K. (2013). Composições cromáticas no ambiente hospitalar: estudo de novas abordagens. Faculdade de Arquitetura e Urbanismo, 215.

McEwen, M., \& Wills, E. M. (2009). Grandes teorias da enfermagem baseadas nas necessidades humanas. Em M. McEwen, \& E. M. Wills, McEwen, M; Wills, E M; (pp. 156-85). Artmed.

Ministério da Saúde. (12 de maio de 1998). PORTARIA $N^{o}$ 2616, de 12 de maio de 1998. http://bvsms.saude.gov.br/: http://bvsms.saude.gov.br/bvs/saudelegis/gm/1998/prt2616_12_05_1998.html

Ministério da Saúde. (21 de Fevereiro de 2002). Resolução de Diretoria Colegiada (RDC) $n^{\circ}$ 50, de 21 de fevereiro de 2002. https://bvsms.saude.gov.br: https://bvsms.saude.gov.br/bvs/saudelegis/anvisa/2002/rdc0050_21_02_2002.html

Nelson, S., \& Gordon, S. (2006). The Complexities of Care: Nursing Reconsidered. Cornell University Press.

Padilha, M. I. (2020). De Florence Nightingale à Pandemia Covid-19. Texto \& Contexto - Enfermagem. https://doi.org/10.1590/1980-265x-tce-2020-0327

Pereira, A. S., Shitsuka, D., Parreira, F., \& Shitsuka, R. (2018). Metodologia da pesquisa científica [recurso eletrônico]. ufsm.b: https://www.ufsm.br/app/uploads/sites/358/2019/02/Metodologia-da-Pesquisa-Cientifica_final.pdf 